\title{
Flavonoid (myricetin, quercetin, kaempferol, luteolin, and apigenin) content of edible tropical plants
}

\begin{abstract}
Studies were conducted on the flavonoids (myricetin, quercetin, kaempferol, luteolin, and apigenin) contents of 62 edible tropical plants. The highest total flavonoids content was in onion leaves (1497.5 mg/kg quercetin, $391.0 \mathrm{mg} / \mathrm{kg}$ luteolin, and $832.0 \mathrm{mg} / \mathrm{kg} \mathrm{kaempferol}$ ), followed by Semambu leaves $(2041.0 \mathrm{mg} / \mathrm{kg})$, bird chili $(1663.0 \mathrm{mg} / \mathrm{kg})$, black tea $(1491.0$ $\mathrm{mg} / \mathrm{kg})$, papaya shoots $(1264.0 \mathrm{mg} / \mathrm{kg})$, and guava $(1128.5 \mathrm{mg} / \mathrm{kg})$. The major flavonoid in these plant extracts is quercetin, followed by myricetin and kaempferol. Luteolin could be detected only in broccoli $(74.5 \mathrm{mg} / \mathrm{kg}$ dry weight), green chili $(33.0 \mathrm{mg} / \mathrm{kg})$, bird chili $(1035.0 \mathrm{mg} / \mathrm{kg})$, onion leaves $(391.0 \mathrm{mg} / \mathrm{kg})$, belimbi fruit $(202.0 \mathrm{mg} / \mathrm{kg})$, belimbi leaves $(464.5 \mathrm{mg} / \mathrm{kg})$, French bean $(11.0 \mathrm{mg} / \mathrm{kg})$, carrot $(37.5 \mathrm{mg} / \mathrm{kg})$, white radish $(9.0 \mathrm{mg} / \mathrm{kg})$, local celery $(80.5 \mathrm{mg} / \mathrm{kg})$, limau purut leaves $(30.5 \mathrm{mg} / \mathrm{kg})$, and dried asam gelugur $(107.5$ $\mathrm{mg} / \mathrm{kg})$. Apigenin was found only in Chinese cabbage $(187.0 \mathrm{mg} / \mathrm{kg})$, bell pepper $(272.0$ $\mathrm{mg} / \mathrm{kg})$, garlic $(217.0 \mathrm{mg} / \mathrm{kg})$, belimbi fruit $(458.0 \mathrm{mg} / \mathrm{kg})$, French peas $(176.0 \mathrm{mg} / \mathrm{kg})$, snake gourd $(42.4 \mathrm{mg} / \mathrm{kg})$, guava $(579.0 \mathrm{mg} / \mathrm{kg})$, wolfberry leaves $(547.0 \mathrm{mg} / \mathrm{kg})$, local celery $(338.5 \mathrm{mg} / \mathrm{kg})$, daun turi $(39.5 \mathrm{mg} / \mathrm{kg})$, and kadok $(34.5 \mathrm{mg} / \mathrm{kg})$. In vegetables, quercetin glycosides predominate, but glycosides of kaempferol, luteolin, and apigenin are also present. Fruits contain almost exclusively quercetin glycosides, whereas kaempferol and myricetin glycosides are found only in trace quantities.
\end{abstract}

Keyword: Flavonoids; Edible tropical plants; Myricetin; Quercetin; Kaempferol; Luteolin; Apigenin 